\title{
Anesthesia and ultrasound: riding the waves
}

\author{
A. Stéphane Lambert, MD · Claude P. Tousignant, MD
}

Received: 15 October 2012/ Accepted: 22 October 2012/Published online: 8 December 2012

(C) Canadian Anesthesiologists' Society 2012

Esophageal echocardiography was first described in $1976^{1}$ and it wasn't long before it was introduced into anesthesia practice. In the mid-eighties, Smith et al. reported that regional wall motion abnormalities observed on TEE, were more sensitive than ECG monitoring in the detection of intraoperative myocardial ischemia. ${ }^{2}$ This led to an explosion in the use of intraoperative TEE monitoring across North America. Transesophageal echocardiography elevated the thinking of cardiac anesthesiologists to new levels, from a deeper understanding of mitral valve anatomy to the subtle and complex nature of right ventricular dysfunction and the intricacies of diastolic dysfunction.

The evolution of intraoperative TEE was not without challenges. Many deplored the uneven adherence to practice standards amongst anesthesiologists. The controversy reached a peak in 1999 when Stevenson reported a sharp increase in the number of "misdiagnoses" in pediatric congenital cardiac surgery patients when anesthesiologists did not follow practice guidelines. ${ }^{3}$ This led to a heated debate about whether anesthesiologists should use TEE at all, and how the use of TEE by insufficiently trained anesthesiologists was bound to have an adverse effect on patient outcomes. ${ }^{4}$ Ultimately, the consensus was that the clinician's title (anesthesiologist $v \mathrm{~s}$ cardiologist) mattered little, provided that competence and adherence to established guidelines were assured.

\footnotetext{
A. S. Lambert, MD ( $\square)$

Division of Cardiac Anesthesiology, University of Ottawa Heart Institute, University of Ottawa, 40 Ruskin Street, Ottawa,

ON K1Y 4W7, Canada

e-mail: slambert@ottawaheart.ca

C. P. Tousignant, MD

Department of Anesthesiology, St. Michael's Hospital, University of Toronto, Toronto, ON, Canada
}

The need for regulation led the American Society of Echocardiography in cooperation with the Society of Cardiovascular Anesthesiologists to publish guidelines for the training and maintenance of competence in perioperative $\mathrm{TEE}^{5}$ and to introduce guidelines for the performance of a comprehensive TEE exam - the so-called "basic exam". ${ }^{6}$ A set of Canadian guidelines soon followed. ${ }^{7}$ Finally, a formal certification process was created for perioperative TEE.

In the end, TEE has emerged as the most important development in cardiac anesthesia in the past 40 years, and arguably, it has redefined the role of the cardiac anesthesiologist. Not only do we continue to be facilitators in the operating room, but we now take an active role in the surgical decision making process, which ultimately has an important and positive impact on patient outcome.

Because ultrasound is such a powerful tool, it has found numerous applications outside of cardiac imaging, such as central catheter insertion and regional anesthesia. Transesophageal echocardiography has also become an invaluable tool outside of cardiac anesthesia, from monitoring high-risk cardiac patients undergoing non-cardiac surgery to the diagnosis of unexplained hemodynamic instability in the intensive care unit and operating room. However, because it is invasive, TEE remains difficult to apply in the ambulatory setting, such as in preoperative clinics or in extubated critically ill patients.

Transthoracic echocardiography (TTE) and general surface ultrasound have begun to blossom in areas like critical care medicine, emergency medicine, and even in preoperative clinics. ${ }^{8}$ At the present time, the expectations and goals of such diverse applications of ultrasound imaging remain relatively simple and focused. "Competency" results from short courses in ultrasound applications that are focused mainly on pleural, thoracic, abdominal, and vascular examinations. ${ }^{9}$ Nevertheless, studies have shown that a 
reasonable degree of competency can be achieved through such courses. ${ }^{9-11}$

Certain institutions have also developed short courses in cardiac imaging specifically. Such basic ultrasound courses instil a sense of confidence, yet the ultrasonographer often behaves like a technician concerned mostly with image acquisition. Examinations are reduced to pattern recognition or yes/no scenarios. However, while image acquisition is relatively simple when using a simulator or in thin healthy volunteers, we know that it is often much more challenging in clinical practice. The link between cardiac physiology and acquired images only comes with experience. For example, not all pericardial collections are associated with demonstrable tamponade physiology.

The use of TTE outside of traditional echo labs expands yet further. Anesthesiologists are moving beyond the urgent needs of critically ill or unstable patients to the elective environment of pre-anesthetic clinics. ${ }^{8}$ They are using echocardiography to enhance physical examinations. All the troublesome illdefined murmurs and dyspnea on exertion, which used to result in surgical delays, can now be evaluated on the spot by the anesthesiologist. The rationale for this increase in scope of practice is the understanding that more information should result in a safer anesthetic. Indeed, an audit of TTEs performed over a nine-month period in the operating room and preoperative clinic showed a high incidence of previously undetected abnormalities, many of which led to changes in management and further investigations. ${ }^{8}$ Then again, such a level of expertise cannot be acquired in a 12-hr weekend course.

A panel of international experts recently released a statement on critical care ultrasound in which they attempted to define goals for: (1) general critical care ultrasound, (2) basic critical care echocardiography, and (3) advanced critical care echocardiography. ${ }^{12}$ Certification was not deemed mandatory, but these experts agreed that a structured certification process would be desirable.

What about anesthesia? Should we limit ourselves to the operating room and critical care areas in the same context as when using TEE, or should we expand our use of echocardiography to non-critical care areas, such as preoperative clinics? The quick TTE assessment of a loud murmur in an elderly patient scheduled for hip surgery might minimize delays and lead to a better outcome. How should our role be defined? What are the needs? More importantly, how do we define competency, and how do we ensure the same quality that we deliver in the operating room?

Anesthesiologists are well positioned to translate 20 years of TEE experience to TTE in a limited and well-defined scope of practice. The physics and basic principles of TTE are indeed the same as those in TEE; the images are nearly identical but projected differently, and there is already extensive core knowledge of cardiac physiology in the anesthesia curriculum.
Transthoracic ultrasound can be tricky, however; good images are not as easy to acquire as in TEE, and the learning curve is far flatter. Training and experience are paramount in achieving competency.

In the study by Tanzola et al., ${ }^{13}$ a limited course on transthoracic echocardiography was given to anesthesia residents, and examinations were administered to the residents before and after the course. Targeted didactic sessions did improve the residents' knowledge, as assessed using multiple-choice examinations, including static images and video clips. Is this a valid endpoint? Does this process lead to a meaningful ability to perform echocardiography in clinical practice? Do didactic sessions such as these translate into adequate interpretation skills? Our perioperative experience has taught us that echocardiography is only as good as the person holding the probe, and incorrect information is potentially more dangerous than no information at all. There is no doubt that residents can absorb knowledge through well-designed training sessions; however, our efforts must go far beyond this. We must determine whether the steps that lead to adequate ultrasonography and its interpretation are feasible. That should be the ultimate goal.

Where do we go from here? Tanzola et al. ${ }^{13}$ correctly stated that large-scale investigations will be needed to determine the true impact of a TTE curriculum in anesthesia and whether this increase in knowledge will translate into improved clinical performance and ultimately better patient safety.

Once these facts are established, the dissemination of knowledge must be carefully regulated. The greatest risk of an uncontrolled spread of TTE across anesthesia areas is a decrease in the quality of the diagnostic information and potentially negative outcomes. As was the case with TEE, competence and adherence to established practice standards are paramount.

Various levels of competence should be defined, with training requirements and clear boundaries should be placed on the scope of practice at each level, similar to what exists in critical care. ${ }^{12}$ A didactic curriculum and a minimum number of studies interpreted and personally performed, under strong supervision, will ensure minimum competence and form the basis for future certification. Indeed, the Canadian Emergency Ultrasound Society has developed standards for training as an independent ultrasound practitioner in the emergency department. Following an introductory course, the trainee must perform at least 50 verified scans in a number of relevant areas to assess the quality of image acquisition. This then leads to examination and certification. Structured courses, such as this, could help launch an anesthesia curriculum that could be tiered for necessary skills. 
In 2010, the American Society of Echocardiography released a consensus statement on FOCUS (FOcused Cardiac UltraSound) in the emergency department. ${ }^{14}$ In the introduction lies an important statement: "The principal role for FOCUS is the time-sensitive assessment of the symptomatic patient." This statement should define our starting goals. Close collaboration must be maintained with echo laboratories so that proper consultation can be initiated whenever the observed abnormalities extend beyond the confines of a focused TTE exam.

Finally, an important challenge for anesthesiologists will be to take ownership of the diagnostic information obtained and to ensure proper follow-up. Indeed, anesthesiologists specialize in acute perioperative medicine, but they lack the benefit of long-term relationships with patients. Any pathology identified perioperatively will require proper referral for further workup, treatment and follow-up.

After the intensive care unit and the emergency room, the pre-anesthetic clinic is the next frontier of point-of-care diagnostic ultrasound. The judicious use of TTE may reduce preoperative delays and improve efficiency and quality of care. In that context, TTE falls clearly within the realm of expert perioperative physicians. However, we must set high standards, so we can deliver TTE with the same quality that we hold ourselves to in the operating room.

\section{Anesthésie et échoguidage: surfons sur la vague}

L'échocardiographie œsophagienne a été décrite pour la première fois en $1976 .{ }^{1}$ Il n'a fallu que peu de temps avant qu'elle ne soit introduite dans la pratique de l'anesthésie. Au milieu des années 1980, Smith et coll. rapportaient que les anomalies régionales de la contractilité des parois observées par échocardiographie transœsophagienne (ÉTO) étaient plus sensibles que le monitorage ECG pour dépister l'ischémie myocardique peropératoire. ${ }^{2}$ Ceci a entrâné une explosion dans l'utilisation du monitorage peropératoire par ÉTO en Amérique du Nord. L'échocardiographie transœsophagienne a élevé la pensée des anesthésiologistes cardiaques vers de nouveaux sommets, qu'il s'agisse d'une compréhension plus approfondie de l'anatomie de la valve mitrale ou de la nature à la fois subtile et complexe du dysfonctionnement du ventricule droit et des subtilités de la dysfonction diastolique.

L'évolution de l'ÉTO peropératoire ne s'est pas faite sans heurts. Nombreux sont ceux qui ont déploré le respect inégal des normes de pratique par les anesthésiologistes. La controverse a atteint un sommet en 1999 lorsque Stevenson a rapporté une augmentation brutale du nombre de « mauvais diagnostics » chez les enfants subissant une chirurgie cardiaque pour anomalies congénitales, lorsque les anesthésio logistes ne suivaient pas les directives de pratique. ${ }^{3}$ Ceci a mené à un débat animé quant à savoir si les anesthésiologistes devaient utiliser l'ÉTO ou non, et quant à la façon dont l'utilisation de l'ÉTO par des anesthésiologistes insuffisamment formés devait nécessairement avoir un impact négatif sur le pronostic des patients. ${ }^{4}$ Finalement, le consensus a été que le titre (anesthésiologiste vs. cardiologue) importait peu, pourvu que la compétence et le respect des directives établies soient garantis.

Le besoin de réglementation a conduit la Société américaine d'échocardiographie (American Society of Echocardiography) et la Société des anesthésiologistes cardiovasculaires (Society of Cardiovascular Anesthesiologists) à publier conjointement des directives concernant la formation et le maintien des compétences en ÉTO périopératoire ${ }^{5}$ ainsi qu'à introduire des directives concernant la réalisation d'un examen d'ÉTO complet -l'examen dit « de base $»{ }^{6}$ Un ensemble de directives canadiennes a suivi peu après. ${ }^{7}$ Par la suite, un processus formel d'accréditation a été créé pour l'ÉTO périopératoire.

En fin de compte, l'ÉTO constitue le développement le plus important en anesthésie cardiaque au cours des 40 dernières années et elle a possiblement redéfini le rôle de l'anesthésiologiste cardiaque. Non seulement nous continuons de jouer le rôle de facilitateur dans la salle d'opération, mais en plus nous jouons désormais également un rôle actif dans le processus de décision chirurgicale, ce qui a, en bout de ligne, un impact important et positif sur le pronostic des patients.

L'échoguidage étant un outil tellement puissant, nous lui avons trouvé de nombreuses applications en dehors de l'imagerie cardiaque, comme par exemple pour l'insertion des cathéters centraux et pour l'anesthésie régionale. L'échocardiographie transœsophagienne est également devenue un outil précieux en dehors de l'anesthésie cardiaque, qu'il s'agisse de surveiller les patients avec pathologie cardiaque grave subissant une chirurgie non cardiaque ou de poser un diagnostic concernant une instabilité hémodynamique inexpliquée à l'unité des soins intensifs et en salle d'opération. Toutefois, en raison de son aspect invasif, il reste difficile d'utiliser l'ÉTO dans un contexte ambulatoire, comme par exemple dans les cliniques préopératoires ou chez les patients extubés mais instables.

L'échocardiographie transthoracique (ÉTT) et l'ultrasonographie de surface en général ont fait leur apparition dans des domaines tels que la médecine des soins critiques, la médecine d'urgence, et même dans les cliniques préopératoires. ${ }^{8}$ À l'heure actuelle, les attentes et les objectifs d'applications aussi variées de l'échographie demeurent relativement simples et ciblés. La « compétence » est le résultat de brefs cours portant sur les applications de l'échographie qui se concentrent principalement sur les examens des poumons, du thorax, de l'abdomen et des 
vaisseaux. ${ }^{9}$ Des études ont toutefois démontré qu'un niveau raisonnable de compétence peut être atteint grâce à de tels cours. ${ }^{9-11}$

Certaines institutions ont également mis au point des cours de courte durée spécifiquement consacrés à l'imagerie cardiaque. De tels cours d'échoguidage de base instillent un sentiment de confiance, mais l'échographiste se comporte souvent comme un technicien dont le rôle principal est d'acquérir des images. Les examens sont réduits à une reconnaissance de schémas et des scénarios oui/non. Toutefois, alors que l'acquisition d'images est relativement simple sur un simulateur ou chez des volontaires sains et minces, nous savons que c'est souvent bien plus difficile dans la pratique clinique. La capacité de faire un lien entre la physiologie cardiaque et les images acquises ne vient qu'avec l'expérience. Par exemple, tous les épanchements péricardiques ne sont pas associés à une physiologie démontrable de tamponnade.

L'utilisation d'ÉTT hors des laboratoires conventionnels d'échographie est encore plus étendue. Les anesthésiologistes vont au-delà des besoins urgents des patients en état critique ou instables en travaillant dans l'environnement non urgent des cliniques préopératoires d'anesthésie. ${ }^{8}$ Ils utilisent l'échocardiographie pour améliorer les examens physiques. Tous les souffles ennuyeux et mal définis de même que la dyspnée à l'effort, qui jusque là engendraient des retards chirurgicaux, peuvent désormais être évalués immédiatement par l'anesthésiologiste. La justification de l'expansion de cette pratique réside dans l'idée que plus on a d'informations, plus l'anesthésie devrait être sécuritaire. En effet, un rapport analysant les ÉTT réalisées au cours d'une période de neuf mois en salle d'opération et dans les cliniques préopératoires a démontré une incidence élevée d'anomalies précédemment non dépistées, ce qui a entraîné des modifications dans la prise en charge et dans les examens complémentaires. ${ }^{8}$ Mais il est évident qu'un tel niveau d'expertise ne peut être atteint en suivant un cours de 12 heures en fin de semaine.

Un panel d'experts internationaux a récemment émis une déclaration concernant l'échographie en soins critiques, dans laquelle ils tentent de définir des objectifs pour: 1) l'ultrasonographie générale dans les soins critiques, 2) l'échocardiographie de base dans les soins critiques, et 3) l'échocardiographie avancée dans les soins critiques. ${ }^{12}$ L'accréditation n'a pas été jugée obligatoire, mais ces experts s'entendent sur le fait qu'un processus d'accréditation structuré serait souhaitable.

Qu'en est-il de l'anesthésie? Devrions-nous nous limiter à la salle d'opération et aux environnements de soins critiques, c'est-à-dire le même contexte que celui dans lequel nous utilisons l'ÉTO? Ou devrions-nous étendre notre utilisation de l'échocardiographie aux environnements hors des soins critiques tels que les cliniques préopératoires?
L'évaluation rapide par ÉTT d'un souffle prononcé chez un patient devant subir une chirurgie de la hanche pourrait minimiser les retards et entraîner un meilleur pronostic. Comment notre rôle devrait-il être défini? Quels sont les besoins? Et, plus important encore, comment définissons-nous la compétence et comment garantissons-nous la même qualité que celle que nous offrons en salle d'opération?

Les anesthésiologistes occupent une excellente position pour traduire 20 ans d'expérience en ÉTO à l'ÉTT, si celle-ci s'inscrit dans un champ de pratique limité et bien défini. La physique et les principes de bases de l'ÉTT sont en effet les mêmes que ceux de l'ÉTO; les images sont quasiment identiques, bien que projetées différemment, et des connaissances fondamentales étendues de la physiologie cardiaque sont déjà couvertes par la formation en anesthésie.

L'échographie transthoracique peut être délicate; il n'est pas aussi facile d'acquérir de bonnes images qu'avec l'ÉTO et la courbe d'apprentissage est bien plus aplatie. La formation et l'expérience sont cruciales pour acquérir le niveau de compétence nécessaire.

Dans une étude de Tanzola et coll., ${ }^{13}$ un cours limité sur l'échocardiographie transthoracique a été donné à des résidents en anesthésie qui ont dû passer des examens avant et après le cours. Les séances didactiques ciblées ont amélioré les connaissances des résidents telles qu'évaluées par des examens à choix de réponse, lesquels comprenaient des images statiques et des capsules vidéo. Ce critère d'évaluation est-il valable? Ce processus entraîne-t-il une véritable capacité à réaliser une échocardiographie dans la pratique clinique? Les séances didactiques telles que celles décrites dans cette étude se traduisent-elles dans des compétences d'interprétation adéquates? Notre expérience dans le contexte périopératoire nous a appris que l'échocardiographie ne sera jamais meilleure que la personne qui tient la sonde et que des informations erronées peuvent être plus dangereuses que le manque d'informations. Il ne fait aucun doute que les résidents peuvent acquérir des connaissances dans le cadre de séances de formation bien conçues. Toutefois, nos efforts doivent aller bien au-delà. Nous devons déterminer si les étapes qui mènent à une échographie et à son interprétation adéquates sont réalisables. Voilà l'objectif ultime.

Et maintenant, où allons-nous? Tanzola et coll. ${ }^{13}$ ont à juste titre déclaré que des études d'envergure seront nécessaires afin de déterminer le véritable impact d'un cours en ÉTT en anesthésie et si ces connaissances supplémentaires se traduiront par une meilleure performance clinique, laquelle résulterait en une sécurité améliorée pour les patients.

Une fois cela établi, la diffusion des connaissances doit être réglementée avec soin. Le plus grand risque lié à une diffusion non contrôlée de l'ÉTT dans tous les domaines de l'anesthésie serait une réduction de la qualité des 
informations diagnostiques, entraînant potentiellement des pronostics négatifs. Tout comme ce fut le cas avec l'ÉTO, la compétence et le respect de normes de pratique établies sont cruciaux.

Différents degrés de compétence devraient être définis, chacun possédant des exigences en matière de formation et des limites claires quant à son champ de pratique, tout comme c'est le cas en soins critiques. ${ }^{12}$ Un programme de cours didactique et un nombre minimal d'examens interprétés et réalisés individuellement, sous une supervision attentive, garantiront l'acquisition des compétences minimales et formeront la base d'une accréditation future. La Société canadienne d'échographie au département d'urgence a en effet mis au point des normes de formation exigées afin de devenir un échographiste indépendant au département des urgences. Après un cours d'introduction, la personne en formation doit réaliser au moins 50 images d'échographie vérifiées et ce, dans plusieurs domaines pertinents afin d'évaluer la qualité des images acquises. Puis viennent l'examen et l'accréditation. Des cours structurés tels que celui-ci pourraient servir de base pour lancer un programme de cours en anesthésie, lequel pourrait comporter plusieurs niveaux selon les compétences nécessaires.

En 2010, la Société américaine d'échocardiographie (American Society of Echocardiography) a émis une déclaration de consensus sur l'échographie cardiaque ciblée, ou FOCUS pour son acronyme anglais (FOcused Cardiac UltraSound), au département des urgences. ${ }^{14}$ L'introduction comporte une déclaration importante: «Le rôle principal de FOCUS est l'évaluation en temps opportun du patient symptomatique. » Cette déclaration devrait définir nos objectifs de départ. Une étroite collaboration doit être maintenue avec les laboratoires d'échographie afin qu'une consultation opportune puisse avoir lieu dès que les anomalies observées vont au-delà des limites d'un examen ciblé d'ÉTT.

Enfin, un défi important pour les anesthésiologistes sera de s'approprier les informations diagnostiques obtenues et de garantir un suivi adapté: en effet, les anesthésiologistes sont des spécialistes de la médecine périopératoire aiguë, mais ils n'ont pas l'avantage des relations à long terme avec leurs patients. Toute pathologie identifiée dans le cadre périopératoire devra être adéquatement acheminée pour des examens complémentaires, un traitement et un suivi.

Après l'unité des soins intensifs et la salle des urgences, la clinique préopératoire d'anesthésie constitue la prochaine frontière de l'imagerie diagnostique au chevet du patient. L'utilisation judicieuse de l'ÉTT pourrait réduire les retards préopératoires et améliorer l'efficacité et la qualité des soins. Dans un tel contexte, l'ÉTT tombe clairement dans le domaine des médecins périopératoires experts. Nous devons toutefois établir des normes élevées afin que nous puissions offrir l'ÉTT avec la même qualité que celle que nous offrons en salle d'opération.
Conflicts of interest None declared.

\section{References}

1. Frazin L, Talano JV, Stephanides L, Loeb HS, Kopel L, Gunnar RM. Esophageal echocardiography. Circulation 1976; 54: 102-8.

2. Smith JS, Cahalan MK, Benefiel DJ, et al. Intraoperative detection of myocardial ischemia in high-risk patients: electrocardiography versus two-dimensional transesophageal echocardiography. Circulation 1985; 72: 1015-21.

3. Stevenson JG. Adherence to physician training guidelines for pediatric transesophageal echocardiography affects the outcome of patients undergoing repair of congenital cardiac defects. J Am Soc Echocardiogr 1999; 12: 165-72.

4. Fyfe D. Transesophageal echocardiography guidelines: return to bypass or to bypass the guidelines? J Am Soc Echocardiogr 1999; 12: 343-4.

5. Anonymous. Practice guidelines for perioperative transesophageal echocardiography. A report by the American Society of Anesthesiologists and the Society of Cardiovascular Anesthesiologists Task Force on Transesophageal Echocardiography. Anesthesiology 1996; 84: 986-1006.

6. Shanewise JS, Cheung AT, Aronson S, et al. ASE/SCA guidelines for performing a comprehensive intraoperative multiplane transesophageal echocardiography examination: recommendations of the American Society of Echocardiography Council for Intraoperative Echocardiography and the Society of Cardiovascular Anesthesiologists Task Force for Certification in Perioperative Transesophageal Echocardiography. Anesth Analg 1999; 89: 870-84.

7. Cardiovascular Section of the Canadian Anesthesiologists' Society; Canadian Society of Echocardiography; Beique F, Ali M, Hynes $M$, et al. Canadian guidelines for training in adult perioperative transesophageal echocardiography. Recommendations of the Cardiovascular Section of the Canadian Anesthesiologists' Society and the Canadian Society of Echocardiography. Can J Cardiol 2006; 22: 1015-27.

8. Canty DJ, Royse CF. Audit of anaesthetist-performed echocardiography on perioperative management decisions for noncardiac surgery. Br J Anaesth 2009; 103: 352-8.

9. Jones AE, Tayal VS, Kline JA. Focused training of emergency medicine residents in goal-directed echocardiography: a prospective study. Acad Emerg Med 2003; 10: 1054-8.

10. Vignon P, Mucke F, Bellec F, et al. Basic critical care echocardiography: validation of a curriculum dedicated to noncardiologist residents. Crit Care Med 2011; 39: 636-42.

11. Cowie B, Kluger R. Evaluation of systolic murmurs by transthoracic echocardiography by anesthetic trainees. Anaesthesia 2011; 66: 785-90.

12. Expert Round Table on Ultrasound in ICU. International expert statement on training standards for critical care ultrasonography. Intensive Care Med 2011; 37: 1077-83.

13. Tanzola RC, Walsh S, Hopman WM, Sydor D, Arellano R, Allard $R V$. Brief report: Focused transthoracic echocardiography training in a cohort of Canadian anesthesiology residents: a pilot study. Can J Anesth 2013; 60. doi:10.1007/s12630-012-9811-8

14. Labovitz AJ, Noble VE, Bierig M, et al. Focused cardiac ultrasound in the emergent setting: a consensus statement of the American Society of Echocardiography and American College of Emergency Physicians. J Am Soc Echocardiogr 2010; 23: 1225-30. 